\title{
ON HOMOGENEOUS ALGEBRAS ${ }^{1,2}$
}

\section{MAX KOECHER}

In this report we will sketch a new approach to the theory of finite dimensional nonassociative algebras. Most of the results mentioned here are proved in a joint book with $\mathrm{H}$. Braun [1]. Its intention is to give a satisfying theory for both Jordan algebras and alternative algebras without dealing too much with identities.

1. For simplicity, only finite dimensional algebras $\mathfrak{A}$ with unit element $e$ over a field $\Phi$ of characteristic not 2 shall be considered. For $u \in \mathfrak{A}$ we define the powers of $u$ by $u^{m+1}=u u^{m}$. The left resp. right regular representation $L(u)$ resp. $R(u)$ are given by

$$
u v=L(u) v=R(v) u .
$$

Besides $\mathfrak{A}$ we consider the commutative algebra $\mathfrak{A}^{+}$defined in the same vector space as $\mathfrak{A}$ with the product $u \circ v=(u v+v u) / 2$. The left regular representation of $\mathfrak{Q}^{+}$is $L^{+}(u)=[L(u)+R(u)] / 2$.

By a field extension of a vector space or an algebra $\mathfrak{A}$ we mean any tensor product $\Phi^{\prime} \otimes_{\Phi} \mathfrak{A}$, where $\Phi^{\prime}$ is an extension field of $\Phi$. Let $b_{1}, b_{2}, \cdots, b_{n}$ be a basis of $\mathfrak{A}$ over $\Phi$ and let be $\tau_{1}, \tau_{2}, \cdots, \tau_{n}$ elements algebraically independent over $\Phi$. Putting $\tilde{\Phi}=\Phi\left(\tau_{1}, \tau_{2}, \cdots, \tau_{n}\right)$ we denote by $\tilde{X}=\tilde{\Phi} \otimes_{\Phi} X$ the vector space obtained from the vector space $X$ by extending $\Phi$ to $\tilde{\Phi}$. The element $x=\tau_{1} b_{1}+\tau_{2} b_{2}+\cdots+\tau_{n} b_{n}$ of $\tilde{\mathfrak{A}}$ is called a generic element of $\mathfrak{A}$. Let $X$ be a vector space over $\Phi$ and let $f$ be an arbitrary element in $\tilde{X}$. Then $f$ can be regarded as a rational function $f(x)$ of $x$, because the components of $f$ with respect to a basis of $X$ over $\Phi$ are rational in $\tau_{1}, \tau_{2}, \cdots, \tau_{n}$. Hence $f(x+\tau u)$, $u \in \mathfrak{X}$, is rational in the variable $\tau$. Since $x$ is a generic element, the differential operator

$$
\Delta_{x}^{u} f(x)=\left.\frac{d}{d \tau} f(x+\tau u)\right|_{\tau=0}
$$

is well defined. Moreover it is linear in $u$. This operator satisfies the usual rules for a differential operator.

2. Let $\mathfrak{A}$ be a finite dimensional algebra over the field $\Phi$ with unit element $e$ and let $x$ be a generic element of $\mathfrak{A}$. We call $\mathfrak{A}$ an algebra with inverse if there exists an element $x^{-1} \in \tilde{\mathfrak{A}}$ such that

1 This research was supported in part by NSF-GP-4017.

${ }^{2}$ An address by Professor Koecher delivered before the meeting of the Society at Massachusetts Institute of Technology on October 30, 1965 by invitation of the Committee to Select Hour Speakers for Eastern Sectional Meetings; received by the editors November 29, 1965. 


$$
x x^{-1}=x^{-1} x=e .
$$

Since $L(e)$ resp. $R(e)$ is the identity the determinant of $L(x)$ resp. $R(x)$ is not the zero polynomial. Therefore $x^{-1}$ is uniquely determined by $x$ and is given by $x^{-1}=L^{-1}(x) e=R^{-1}(x) e$. Moreover, $x^{-1}$ is homogeneous in $x$ of degree -1 . Denote by $H N(x), H N(e)=1$, the exact denominator of $x^{-1}$. Then $H N(x)$ is a homogeneous polynomial in $x$ and a divisor of the determinant of $L(x)$ and $R(x)$.

For any field extension $\mathfrak{B}$ of $\mathfrak{A}$ we denote by $I(\mathfrak{B})$ the set of elements $u \in \mathfrak{B}$ such that $u^{-1}$ is defined, i.e. such that $H N(u) \neq 0$. The elements of $I(\mathfrak{B})$ are called invertible. In particular, an element $u \in \mathfrak{B}$ belongs to $I(\mathfrak{B})$ if the determinant of $L(u)$ is different from zero. Note that in general the contrary is not true. If $u \in I(\mathfrak{B})$ we call $u^{-1}$ the inverse of $u$. Given an element $u \in \mathfrak{B}$ one can specialize $x \rightarrow u$ in any polynomial identity involving $x$ and $x^{-1}$. Obviously, any field extension of $\mathfrak{A}$ is an algebra with inverse.

Using $x^{-1} \in I(\tilde{\mathfrak{U}})$ we see that

$$
\left(x^{-1}\right)^{-1}=x
$$

holds. Hence $x^{-1}$ is a generic element of $\mathfrak{A}$, too.

Defining a linear transformation $H(x)$ of $\mathfrak{i}$ by

$$
H(x) u=-\Delta_{x}^{u} x^{-1},
$$

we see that $H(x)$ is rational in $x$ and that $x$ can be specialized to each element $u \in I(\mathfrak{B})$ for any field extension $\mathfrak{B}$ of $\mathfrak{A}$.

Applying $\Delta_{x}^{u}$ on (2) and using the chain rule, we get

$$
H\left(x^{-1}\right) H(x)=I d,
$$

$I d$ is the identity map. The Euler differential equation for the homogeneous rational function $x^{-1}$ leads to

$$
x^{-1}=H(x) x .
$$

Finally, the identities (1) implies

$$
R\left(x^{-1}\right)=L(x) H(x), \quad L\left(x^{-1}\right)=R(x) H(x) .
$$

Thus, in particular, we get $H(e)=I d$ and that $H(x)$ is invertible.

Since any commutative algebra with unit element is an algebra with inverse $x^{-1}=L^{-1}(x) e$, in general any result for those algebras must be trivial in the commutative case.

Lemma 1. Let $\mathfrak{A}$ be an algebra with inverse. Then $(m+1) !\left(x x^{m}-x^{m} x\right)$ $=0$ for any integer $m \geqq 0$. 
Proof. For generic independent elements $x, y$ put $f_{0}(y ; x)=y^{-1}$, and for $m \geqq 1, f_{m}(y ; x)=-\Delta_{u}^{x} f_{m-1}(y ; x)$. Hence $f_{1}(y ; x)=H(y) x$. Applying $\Delta_{y}^{x}$ on $y f_{0}(y ; x)=e=f_{0}(y ; x) y$, we get

$$
x f_{0}(y ; x)=y f_{1}(y ; x) \text { resp. } f_{0}(y ; x) x=f_{1}(y ; x) y .
$$

An induction on $m$ leads to

$$
m x f_{m-1}(y ; x)=y f_{m}(y ; x) \text { resp. } m f_{m-1}(y ; x) x=f_{m}(y ; x) y .
$$

Substituting $e$ for $y$ and putting $x_{m}=f_{m}(e ; x)$, we obtain

$$
x_{m}=m x x_{m-1}=m x_{m-1} x, \quad x_{0}=e, \quad x_{1}=x .
$$

Consequently $x_{m}=m ! x^{m}$ by induction. This proves the lemma.

In the case of characteristic zero, we consequently have $x x^{m}=x^{m} x$.

The commutative algebra $\mathfrak{U}^{+}$has the same inverse $x^{-1}$ as the algebra $\mathfrak{A}$. Consequently, the linear transformation $H^{+}(x)$ for $\mathfrak{U}^{+}$coincides with $H(x)$ for $\mathfrak{A}$ itself. Applying $\Delta_{x}^{u}$ on (5) and substituting $e$ for $x$, we get $H(e ; u)=-L(u)-R(u)$, where $H(x ; u)$ is defined by $H(x ; u)=\Delta_{x}^{u} H(x)$. Hence

$$
L^{+}(u)=-\frac{1}{2} H(e ; u) .
$$

Consequently the algebra $\mathfrak{A}^{+}$is given by the inverse $x^{-1}$.

3. Any linear transformation $W$ of an algebra $\mathfrak{A}$ can be extended to a linear transformation of any field extension of $\mathfrak{A}$. For a generic element $x$ the element $W x$ is again generic if $W$ is invertible.

Let $\mathfrak{A}$ be an algebra with inverse. Denote by $\Gamma(\mathfrak{U})$ the set of invertible transformations $W: \mathfrak{U} \rightarrow \mathfrak{A}$ for which there exists an invertible linear transformation $W^{\#}: \mathfrak{A} \rightarrow \mathfrak{A}$ such that

$$
(W x)^{-1}=W^{\sharp-1} x^{-1} \text {. }
$$

Applying $\Delta_{x}^{u}$ on this identity and using the chain rule we get

$$
W^{\#} H(W x) W=H(x) \text {. }
$$

Conversely, (7) follows from $\left(7^{\prime}\right)$ using (4). Hence the conditions (7) and $\left(7^{\prime}\right)$ are equivalent. In particular, $W^{\#}$ is uniquely determined by $W$. It is easy to see that $\Gamma(\mathfrak{U})$ is a group and moreover that $W \rightarrow W^{\#}$ is an anti-homomorphism of $\Gamma(\mathfrak{U})$. We call $\Gamma(\mathfrak{U})$ the structure group of $\mathfrak{A}$. Since $x^{-1}$ is a generic element too, we may replace $x$ by $x^{-1}$ and $W$ by $W^{-1}$ in (7). Then the formula (2) yields $W^{\#} \in \Gamma(\mathfrak{U})$ and $\left(W^{\sharp}\right)^{\sharp}=W$ for $W \in \Gamma(\mathfrak{A})$.

From (7) we conclude that $H N(W x) x^{-1}$ is a polynomial for $W \in \Gamma(\mathfrak{U})$. Since $H N(x)$ is the exact denominator of $x^{-1}$ we see that $H N(x)$ is a divisor of $H N(W x)$. But both polynomials have the same degree. Hence 


$$
H N(W x)=\kappa(W) H N(x), \quad W \in \Gamma(\mathfrak{A}),
$$

and $\kappa(W)$ belongs to the ground field. In particular, for $\mathfrak{B}$ a field extension of $\mathfrak{A}$, any element $W \in \Gamma(\mathfrak{B})$ maps $I(\mathfrak{B})$ onto itself.

In order to show that the elements of $\Gamma(A)$ can be defined by polynomial identities we consider the element $p(x)=H N(x) x^{-1}$ of $\mathfrak{A}$. Since $H N(x)$ is the denominator of $x^{-1}$ the elements $p(x)$ and $[H N(x)]^{2} H(x)$ are polynomials in $x$.

Let $Z$ be a generic element of the vector space Hom $\mathfrak{A}$ and let

$$
\pi(Z)=H N(Z e), \quad Q(Z)=\pi^{2}(Z) H(Z e) Z .
$$

Then $\pi$ and $Q$ are polynomials in $Z$. From $\left(7^{\prime}\right)$ and (8) follows $\pi(W)=\kappa(W)$ and $W^{\sharp} Q(W)=\pi^{2}(W) I d$, whenever $W \in \Gamma(\mathfrak{X})$. Hence

$$
\pi(W) p(W x)=Q(W) p(x), \quad H N(W x)=\pi(W) H N(x),
$$

whenever $W \in \Gamma(\mathfrak{U})$. Vice versa, let $W$ be an invertible linear transformation satisfying $\left(7^{\prime \prime}\right)$. Then $\pi(W) \neq 0$ and the transformation $Q(W)$ is invertible. Defining $W^{\#}=\pi^{2}(W) Q^{-1}(W)$ we see that $(7)$ is a consequence of $\left(7^{\prime \prime}\right)$. Therefore an invertible linear transformation $W$ belongs to $\Gamma(\mathfrak{H})$ if and only if $\left(7^{\prime \prime}\right)$ is satisfied.

If the field $\Phi$ is infinite, then the identities $\left(7^{\prime \prime}\right)$ can be replaced by conditions $\pi_{i}(W)=0$ where $\pi_{i}$ are polynomials in the generic element $Z$ with coefficients in $\Phi$. Hence $\Gamma(\mathfrak{U})$ is a linear algebraic group defined over $\Phi$, whenever $\Phi$ is infinite.

Since the inverses of $\mathfrak{U}$ and $\mathfrak{H}^{+}$coincide, we have $\Gamma(\mathfrak{U})=\Gamma\left(\mathfrak{U}^{+}\right)$.

Let $V$ be an automorphism of the algebra $\mathfrak{A}$. Then $V$ is an automorphism of $\tilde{\mathfrak{A}}$ also. Consequently we get $(V x)^{-1}=V x^{-1}$, because the inverse of a generic element is uniquely determined. Therefore any automorphism of $\mathfrak{A}$ belongs to $\Gamma(\mathfrak{U})$.

Lemma 2. Let $\mathfrak{A}$ be a commutative algebra with unit element e. Then a linear transformation $V$ of $\mathfrak{A}$ is an automorphism of $\mathfrak{A}$ if and only if $V \in \Gamma(\mathfrak{U})$ and $V e=e$. In this case $V^{*}=V^{-1}$.

Note that any commutative algebra with unit element is an algebra with inverse.

Proof. We know already that the statement is true if $V$ is an automorphism of $\mathfrak{A}$. Conversely let $V \in \Gamma(\mathfrak{H})$ and $V e=e$. We may conclude $V^{\sharp}=V^{-1}$ by substituting $e$ for $x$ in $\left(7^{\prime}\right)$. Applying $\Delta_{x}^{u}$ on $\left(7^{\prime}\right)$ the same arguments leads to $V^{*} H(e ; V u) V=H(e ; u)$. Hence, from (6) we get $L(V u) V=V L(u)$, and consequently $V$ is an automorphism of $\mathfrak{A}$.

For an algebra with inverse, denote by $I_{0}(\mathfrak{A})$ the orbit of the unit element $e$ under $\Gamma(\mathfrak{I})$, that is, let $I_{0}(\mathfrak{H})=\{W e ; W \in \Gamma(\mathfrak{U})\}$. Using 
$\left(7^{\prime}\right)$ and $W^{*} \in \Gamma(\mathfrak{R})$ for $W \in \Gamma(\mathfrak{H})$, we see that

$$
H(u) \in \Gamma(\mathfrak{I}) \quad \text { and } \quad H^{\sharp}(u)=H(u) \quad \text { for } u \in I_{0}(\mathfrak{I}) .
$$

Consequently (7) and ( $\left.7^{\prime}\right)$ leads to

(9) $[H(u) x]^{-1}=H^{-1}(u) x, H(u) H(H(u) x) H(u)=H(x)$ for $u \in I_{0}(\mathfrak{A})$.

There is a natural algebraic structure on the set $I_{0}(\mathfrak{H})$ : We define a composition $u \cdot v$ for $u, v \in I_{0}(\mathfrak{I})$ by

$$
u \cdot v=H^{-1}(u) v^{-1} .
$$

This is well defined because $I_{0}(\mathfrak{Y}) \subset I(\mathfrak{Y})$. An easy calculation using (2), (3), (4) and (9) leads to

$$
\begin{aligned}
u \cdot u & =u, \\
u \cdot(u \cdot v) & =v, \\
u \cdot(v \cdot w) & =(u \cdot v) \cdot(u \cdot w) .
\end{aligned}
$$

In general, the map $v \rightarrow u \cdot v$ is not an injection of $I_{0}(A)$. Following $O$. Loos we call a set having a composition $u \cdot v$ a reflection space, if the composition fulfills (R.i)-(R.iii). Using (7) and ( $\left.7^{\prime}\right)$ we calculate $W(u \cdot v)=W u \cdot W v$ for $W \in \Gamma(\mathfrak{R})$. Consequently, $\Gamma(\mathfrak{I})$ is a group of automorphism of the reflection space $I_{0}(\mathfrak{Q})$.

4. Let $\mathfrak{A}$ be an algebra over $\Phi$ with unit element $e$ satisfying $x x^{2}=x^{2} x$. By linearization we find that in

(10) $P(x)=L(x)[L(x)+R(x)]-L\left(x^{2}\right)=R(x)[L(x)+R(x)]-R\left(x^{2}\right)$

both expressions define the same transformation $P(x)$. Equivalent with this definition is

$$
P(x) y=x(x y+y x)-x^{2} y=(x y+y x) x-y x^{2} .
$$

In particular, we have $P(e)=I d$. We call $P$ the quadratic representation of $\mathfrak{A}$. We conclude from (10) that the quadratic representation of $\mathfrak{A}$ and of $\mathfrak{Q}{ }^{+}$coincide.

An algebra $\mathfrak{A}$ with inverse is called homogeneous, if, for any field extension $\mathfrak{B}$ of $\mathfrak{A}$ having algebraically closed ground field, the group $\Gamma(\mathfrak{B})$ acts transitively on the set $I(\mathfrak{B})$, the set of invertible elements of $\mathfrak{B}$.

THEOREM 1. Let $\mathfrak{A}$ be an algebra with inverse (satisfying $x^{2} x=x x^{2}$ in case of characteristic 3 ). Then the following statements are mutually equivalent:

(i) $\mathfrak{A}$ is homogeneous.

(ii) $[H(y) x]^{-1}=H^{-1}(y) x^{-1}, x$ and $y$ being generically independent. 
(iii) $H(u) \in \Gamma(\mathfrak{B})$ and $H^{\sharp}(u)=H(u)$, for any invertible $u \in \mathfrak{B}, \mathfrak{B}$ any field extension of $\mathfrak{A}$.

(iv) $\mathfrak{H}^{+}$is a Jordan algebra.

Each of these statements implies that $H^{-1}(x)$ coincides with the quadratic representation $P(x)$ of $\mathfrak{A}$.

Proof. Note first, that $x^{2} x=x x^{2}$ is satisfied (see Lemma 1) and consequently $P(x)$ is well defined. We may assume that $\mathfrak{A}$ is commutative.

The equivalence of (ii) and (iii) follows immediately from the definition of $\Gamma(\mathfrak{H})$ and the fact that in $H(y)$ the generic element $y$ can be specialized to any invertible element $u \in \mathfrak{B}$.

In order to show that (iii) is a consequence of (i), we may assume that $u$ is a invertible element of $\mathfrak{B}$ and that $\mathfrak{B}$ has an algebraically closed ground field. Choosing $W \in \Gamma(\mathfrak{B})$ such that $u=W e$ we see from $\left(7^{\prime}\right)$ that $H(u)=H(W e)=W^{\#} W \in \Gamma(\mathfrak{B})$, because $W^{\#}$ belongs to $\Gamma(\mathfrak{B})$ also.

Next we use the fact that for commutative algebras (ii),

(v) $H(x)$ and $L(x)$ commute,

(vi) $L(x)$ and $L\left(x^{-1}\right)$ commute,

are mutually equivalent and each of these statements implies $H^{-1}(x)$ $=P(x)$. This is proved in part (c) of the Equivalence-Lemma in $[1$, p. 67], for strictly power associative algebras. Checking the proof we see, that it works for commutative algebras because (v) and (vi) imply $x^{2} x^{-1}=x$.

Consequently (ii) implies (v) and $H^{-1}(x)=P(x)$. Therefore $P(x)$ and $L(x)$ commute and hence $L\left(x^{2}\right)$ and $L(x)$ commute. Thus $\mathfrak{A}$ is a Jordan algebra.

Finally let $\mathfrak{A}$ be a Jordan algebra. Then $L(x)$ and $L\left(x^{-1}\right)$ commute. Consequently (ii) and therefore (iii) and $H^{-1}(x)=P(x)$ hold. It follows that $P(u)$ belongs to $\Gamma(\mathfrak{B})$ for any invertible element $u \in \mathfrak{B}$, where $\mathfrak{B}$ is any field extension of $\mathfrak{A}$. Now let $\mathfrak{B}$ be a field extension of $\mathfrak{A}$ having algebraically closed ground field and let $v$ be an invertible element of $\mathfrak{B}$. We choose an invertible element $u \in \mathfrak{B}$ such that $v=u^{2}$ (see [1, Chapter I, Theorem 4.3]). Hence $P(u) e=u^{2}=v$ and $\Gamma(\mathfrak{B})$ acts transitively on $I(\mathfrak{B})$.

REMARK. Only straight forward results on Jordan algebras are used for the proof of this theorem.

From (iii) and $H^{-1}(x)=P(x)$ we obtain

Corollary 1. Let $\mathfrak{A}$ be a homogeneous algebra. Then $P(P(y) x)$ $=P(y) P(x) P(y)$ for generically independent $x$ and $y$.

and 
Corollary 2. Let $\mathfrak{A}$ be a homogeneous algebra. Then $P(u)$ belongs to $\Gamma(\mathfrak{H})$ whenever $u \in \mathfrak{A}$ is invertible.

Using the well known result that a flexible algebra is a noncommutative Jordan algebra if and only if $\mathfrak{Q}^{+}$is Jordan we get

Corollary 3. Let $\mathfrak{A}$ be a flexible algebra. Then $\mathfrak{A}$ is homogeneous if and only if $\mathfrak{A}$ is a noncommutative Jordan algebra.

5. We consider now a strictly power associative algebra $\mathfrak{A}$ with unit element. Since the algebra $\Phi[x]$ generated by $x$ is associative and since $x$ is not a zero divisor in $\Phi[x], x$ has an inverse $x^{-1}$ in $\Phi[x]$. Hence $\mathfrak{A}$ is an algebra with inverse.

Let $f(\tau ; x) \in \tilde{\Phi}[\tau]$ be the minimum polynomial of the generic element $x$ of $\mathfrak{A}$. It is wellknown that $f(\tau ; x)$ is also a polynomial in $x$. If $s$ is the degree of $f(\tau ; x)$, then this polynomial and the exact denominator $H N(x)$ of $x^{-1}$ are related as follows:

$$
H N(x)=(-1)^{s} f(0 ; x) .
$$

$H N(x)$ is called the generic norm of $\mathfrak{A}$. Moreover $H N(\tau e-x)$ coincide with the minimum polynomial $f(\tau ; x)$ of $x$. For these results see $\mathrm{N}$. Jacobson [2] and [1, Chapter II].

The polynomial $H N(x)$ has coefficients in the field $\Phi$. Denote by $\omega_{1}(x), \cdots, \omega_{t}(x), \omega_{1}(e)=1$, the different absolutely irreducible (that is, irreducible over the algebraic closure $\bar{\Phi}$ of $\Phi$ ) divisors of $H N(x)$. Putting

$$
R N(x)=\coprod_{i=1}^{t} \omega_{i}(x)
$$

we call $R N(x)$ the reduced norm of $\mathfrak{A}$. Note that, in general, $R N(x)$ has coefficients in $\bar{\Phi}$ and not in $\Phi$.

A homogeneous polynomial $\omega(x)$ in the generic element $x$ of $\mathfrak{A}$ with coefficients in the algebraic closure $\bar{\Phi}$ of $\Phi$ is called multiplicative if $\omega(e)=1$ and if, for any extension field $\Phi^{\prime}$ of $\Phi$,

$$
\omega(u v)=\omega(u) \omega(v) \quad \text { for } u, v \in \Phi^{\prime}[x]
$$

holds. The following theorem gives a description of all multiplicative polynomials:

THEOREM 2. Let $\mathfrak{A}$ be a strictly power associative algebra with unitelement $e$ and let $\omega$ be a polynomial in the generic element $x$ of $\mathfrak{A}$ with coefficients in $\bar{\Phi}$ and which satisfies $\omega(e)=1$. Then $\omega$ is multiplicative if and only if $\omega(x)$ is a monomial in the absolutely irreducible factors $\omega_{i}(x)$ of $H N(x)$. 
Now let $\omega(x)$ be any multiplicative polynomial. Write

$$
\omega(\tau e-x)=\tau^{m}-\chi(x) \tau^{m-1}+-\cdots+(-1)^{m} \omega(x),
$$

then the coefficient $\chi(x)$ is linear in $x$ and its coefficients are in $\bar{\Phi}$. Consequently $\chi(x)$ induces a linear map $\chi: \mathfrak{A} \rightarrow \bar{\Phi}$. One can prove that $\chi$ vanishes for each nilpotent element of any field extension of $\mathfrak{A}$. The linear map associated with $H N(x)$ resp. $R N(x)$ is called the generic trace $H S(x)$ resp. the reduced trace $R S(x)$ of the algebra $\mathfrak{A}$. Note that the coefficients of $H S(x)$ belongs to $\Phi$.

Consider the subgroup $\Lambda(\mathfrak{R})$ of $\Gamma(\mathfrak{I})$ consisting of all $W \in \Gamma(\mathfrak{R})$ such that there exists to any multiplicative polynomial $\omega(x)$ an element $\kappa(W) \in \bar{\Phi}$ with

$$
\omega(W x)=\kappa(W) \omega(x) .
$$

Using (8) one can prove that $\Lambda(\mathfrak{l})$ is an invariant subgroup of finite index in $\Gamma(\mathfrak{H})$. Furthermore we get $P(u) \in \Lambda(\mathfrak{l})$ for any invertible element $u \in \mathfrak{A}$. Using Corollary 2 of Theorem 1 we end with

Lemma 3. Let $\mathfrak{A}$ be a strictly power associative homogeneous algebra and let $\mathfrak{B}$ be a field extension over an algebraically closed field. Then $\Lambda(\mathfrak{B})$ acts transitively on the set $I(\mathfrak{B})$ of invertible elements of $\mathfrak{B}$.

6. Let $\mathfrak{A}$ be a strictly power associative homogeneous algebra and let $\omega(x)$ be a multiplicative polynomial of $\mathfrak{A}$. We introduce a polynomial $\omega(x, y)$ associated with $\omega(x)$ which will play an important role. Using Lemma 3 one can show for generically independent elements $x$ and $y$ that

$$
\omega(x+y)=\omega(x) \omega(y) \omega\left(x^{-1}+y^{-1}\right) .
$$

Replacing $x$ by $x^{-1}$ we see that the rational function

$$
\omega(x, y)=\omega(x) \omega\left(x^{-1}+y\right)
$$

is symmetric in $x$ and $y$. But $\omega(x, y)$ is a polynomial in $y$ and, consequently, $\omega(x, y)$ is a symmetric polynomial in $x$ and $y$.

Using the polynomial $\omega(x, y)$ for the absolutely irreducible factors of $R N(x)$, we are able to prove the following

Theorem 3. Let $\mathfrak{A}$ be a strictly power associative homogeneous algebra and let $x$ be a generic element of $\mathfrak{A}$. Then

(i) $R N(\tau e-x)$ has only simple roots as a polynomial in $\tau$.

(ii) The reduced trace $R S$ is not zero.

For a variable $\tau$ we write

$$
\omega(x, \tau y)=\sum_{j=0}^{m} \alpha_{j}(x, y) \tau^{j}, \quad \alpha_{0}(x, y)=1,
$$


then $\alpha_{j}(x, y)$ is a homogeneous polynomial of degree $j$ in $x$ and in $y$ that is symmetric in $x$ and $y$. Again the definition of $\Lambda(\mathfrak{l})$ yields

$$
\omega(V x, y)=\omega\left(x, V^{\sharp} y\right), \quad V \in \Lambda(\mathfrak{U}) .
$$

Consequently we get

$$
\alpha_{j}(V x, y)=\alpha_{j}\left(x, V^{\sharp} y\right), \quad V \in \Lambda(\mathfrak{R}) .
$$

Since $P(v)$ belongs to $\Lambda(\mathfrak{A})$ and since $P^{\sharp}(v)=P(v)$ whenever $v$ is an invertible element of $\mathfrak{A}$, we conclude that

$$
\alpha_{j}(P(v) u, w)=\alpha_{j}(u, P(v) w),
$$

is true for invertible elements. Hence it is true for arbitrary elements in $\mathfrak{A}$. Linearizing of this identity for $j=1$ leads to

$$
\alpha_{1}(u \circ v, w)=\alpha_{1}(u, v \circ w) \text {. }
$$

Calculating

$$
\omega(\tau e-x)=\tau^{m} \omega\left(e,-\tau^{-1} x\right)=\tau^{m}-\alpha_{1}(e, x) \tau^{m-1}+-\cdots
$$

and comparing this with (11) we see that the linear map $\chi$ associated with the multiplicative polynomial and the coefficient $\alpha_{1}(x, y)$ are related by $\chi(x)=\alpha_{1}(e, x)$. Setting $w=e$ in (13), we obtain

$$
\chi(u \circ v)=\alpha_{1}(u, v) \text {. }
$$

7. Let $\mathfrak{A}$ be an arbitrary algebra over $\Phi$ and let $\lambda$ be a linear map of $\mathfrak{A}$ into an extension field of $\Phi$. The map $\lambda$ is said to be associative if $\lambda(u v)=\lambda(v u), \lambda(u[v w])=\lambda([u v] w)$, holds for $u, v, w \in \mathfrak{A} . \lambda$ is called seminormal if in addition $\lambda$ vanishes on all nilpotent elements of any field extension of $\mathfrak{A}$. Finally $\lambda$ is called normal, if $\lambda$ is seminormal and has values in $\Phi$.

The algebra $\mathfrak{A}$ is said to be nondegenerate, if there exists a normal linear form $\lambda$ of $\mathfrak{A}$ such that the associated bilinear form $\lambda(u v)$ is nondegenerate. It is easy to see that any nondegenerate algebra contains a unit element.

Linearizing the identities $u u^{2}=u^{2} u$ and $u^{2} u^{2}=u^{4}$, and using the associativity of $\lambda$, one can prove

TheOREM 4. Let $\mathfrak{A}$ be a strictly power associative nondegenerate algebra over a field of characteristic not 5 . Then $\mathfrak{A}$ is a noncommutative Jordan algebra.

In the case of homogeneous algebras we get

Theorem 5. Let $\mathfrak{A}$ be a homogeneous nondegenerate algebra. Then the generic norm and the reduced norm coincide. 
8. The results stated in $\S 6$ (in particular (13) and (14)) show that the map $\chi$ associated with the multiplicative polynomial $\omega$ is seminormal for the commutative algebra $\mathfrak{U}^{+}$. We call a strictly power associative homogeneous algebra $\mathfrak{A}$ strongly-homogeneous if for any multiplicative polynomial $\omega$ the map $\chi$ is associative on $\mathfrak{A}$. Obviously in this case $\chi$ is seminormal and it satisfies $\lambda(u v)=\alpha_{1}(u, v)$.

Using this definition we see that any commutative homogeneous algebra (that is any Jordan algebra with unit element) is strongly homogeneous.

The well known result, that for a flexible algebra $\mathfrak{A}$ any linear map $\lambda$ that is associative on $\mathfrak{Q}^{+}$and satisfies $\lambda(u v)=\lambda(v u)$ is associative on $\mathfrak{A}$, leads to the following

Lemma 4. Let $\mathfrak{A}$ be a noncommutative Jordan algebra. Then $\mathfrak{A}$ is strongly-homogeneous if and only if $\chi(u v)=\chi(v u)$ for any multiplicative polynomial $\omega$ of $\mathfrak{A}$.

Part (ii) of Theorem 3 now yields

THEOREM 6. Let $A$ be a strongly-homogeneous algebra. Then the reduced trace $R S$ is nonzero and seminormal.

We give now some further results (for the proofs see [1]).

Theorem 7. Let $\mathfrak{A}$ be a strongly-homogeneous algebra. Then the following statements are equivalent:

(i) The unit element is the unique idempotent of $\mathfrak{A}$.

(ii) Any noninvertible element of $\mathfrak{A}$ is nilpotent.

(iii) The noninvertible elements of $\mathfrak{A}$ form a vector space.

In this case the set of nilpotent elements coincides with the ideal containing all elements $u \in \mathfrak{A}$ such that $R S(u v)=0$ for $v \in \mathfrak{A}$.

As a consequence we see that a nodal noncommutative Jordan algebra is not strongly-homogeneous.

An algebra $\mathfrak{A}$ is called central simple if $\mathfrak{A}$ is simple, contains a unit element $e$ and if the center of $\mathfrak{U}$ coincides with $\Phi e$.

THEOREM 8. Let $\mathfrak{A}$ be a central simple strongly-homogeneous algebra. Then

(i) $\mathfrak{A}$ is nondegenerate with respect to the generic trace.

(ii) The generic norm is absolutely irreducible.

(iii) The group $\Lambda(\mathfrak{I})$ coincides with the structure group $\Gamma(\mathfrak{U})$.

Next, we give another description of the structure group (see [1, Chapter III, Theorem 5.4 and 5.5)].

Theorem 9. Let $\mathfrak{A}$ be a strongly-homogeneous algebra which is non- 
degenerate with respect to the generic trace. Then an invertible linear transformation $W$ of $\mathfrak{A}$ belongs to $\Gamma(\mathfrak{U})$ if and only if there is a $\kappa(W) \in \Phi$ such that $H N(W x)=\kappa(W) H N(x)$. In this case $W^{\#}$ is the adjoint transformation of $W$ with respect to the bilinear form $H S(u v)$.

9. Finally we discuss a sufficient condition for an algebra to be strongly-homogeneous. By analogy with the Corollary 2 of Theorem 1 we consider a noncommutative Jordan algebra $\mathfrak{A}$ for which $L(x)$ belongs to the structure group $\Gamma(\tilde{\mathfrak{H}})$, whenever $x$ is a generic element for $\mathfrak{A}$. Using (8) and Theorem 2 one can show easily that $L(x)$ belongs to $\Lambda(\mathfrak{\mathfrak { I }})$. Now let $\omega$ be a multiplicative polynomial of $\mathfrak{A}$. The definition of $\Lambda(\tilde{\mathfrak{U}})$ leads to

$$
\omega(x, y)=\omega(e+x y),
$$

because $x=L(x) e, x^{-1}+y=L^{-1}(x)(e+x y)$. Consequently $\chi(x y)=\chi(y x)$ and Lemma 4 yields that $\mathfrak{U}$ is strongly-homogeneous.

The hypothesis that $L(x)$ belongs to $\Gamma(\tilde{\mathfrak{A}})$ means $(x y)^{-1}=L^{\#-1}(x) y$. But from $\left(7^{\prime}\right)$ for $x \rightarrow e$ follows that $L^{\#-1}(x)=H(x) L(x)=L(x) H(x)$. Now (5) leads to

$$
L^{\#-1}(x)=R\left(x^{-1}\right),
$$

consequently to $(x y)^{-1}=y^{-1} x^{-1}$, and vice versa. Hence

ThEOREM 10. Any noncommutative Jordan algebra satisfying $(x y)^{-1}$ $=y^{-1} x^{-1}$, where $x, y$ are generically independent elements, is a stronglyhomogeneous algebra.

Since any two elements in an alternative algebra generate an associative algebra we get

Corollary. Any alternative algebra is strongly-homogeneous.

Using (15) and Theorem 9 one can show that any non-degenerate algebra satisfying $(x y)^{-1}=y^{-1} x^{-1}$ is alternative.

\section{REFERENCES}

1. H. Braun and M. Koecher, Jordan-Algebren, Springer-Verlag, Berlin, 1966.

2. N. Jacobson, Generic norm of an algebra, Osaka Math. J. 15 (1963), 25-50.

YALE UNIVERSITY AND

University of Munich, Germany 